\title{
CLOSURE OF PRODUCTS OF FUNCTIONS ${ }^{1}$
}

\author{
D. G. BOURGIN
}

This note presents some natural theorems on the characterizations of certain closed (or complete) sets of functions with separable variables. In order to motivate the developments of the paper we treat a simple case first in elaborate detail. The proof is so formulated that it holds with trifling modifications for the more general situations in Theorems 3 and 4 . The result in Theorem 5 belongs to a slightly different range of ideas.

Let $s \sim\left(s_{1}, \cdots, s_{m}\right)$ and $t \sim\left(t_{1}, \cdots, t_{n}\right)$ here stand for points in the euclidean spaces $R_{m}$ and $R_{n}$. The term "interval" designates the generalized rectangular parallelopipedon open on the left. ${ }^{2} \mathrm{We}$ shall make use of the intervals $I_{s} \subset R_{m}, I_{t} \subset R_{n}$ and $I_{2}=I_{s} \times I_{t} \subset R_{n+m}$. We are first interested in $L_{2}(I)$, the space of complex valued functions of summable square over $I$. The norm and scalar product are defined as usual by

$$
\begin{aligned}
\|f(s, t)-g(s, t)\| & =\left[\int_{I_{t}} \int_{I_{s}}|f(s, t)-g(s, t)|^{2} d I_{s} d I_{t}\right]^{1 / 2}, \\
(f(s, t), g(s, t)) & =\int_{I_{t}} \int_{I_{s}} f(s, t) \bar{g}(s, t) d I_{s} d I_{t},
\end{aligned}
$$

where $\bar{g}(s, t)$ is the conjugate of $g(s, t)$. The subscript $I_{s}$ or $I_{t}$ will indicate that the left-hand functionals are on the corresponding intervals.

We shall understand closure of the sequence of functions ${ }^{3}$ $\left\{\phi_{\gamma}(t) \psi_{\mu}(s)\right\}, \gamma ; \mu=0,1, \cdots$, to mean that for every $f(s, t) \varepsilon L_{2}\left(I_{2}\right)$ and arbitrary $\epsilon>0$ there exists a finite sequence of complex constants $\left\{\beta_{\gamma \mu}\right\}$ and integers $A$ and $B$ such that

$$
\left\|f(s, t)-\sum_{0}^{A} \sum_{0}^{B} \beta_{\gamma \mu} \phi_{\gamma}(t) \psi_{\mu}(s)\right\|<\epsilon .
$$

It is well known that with the adjunction of the scalar product defined in (2), $L_{2}\left(I_{2}\right)$ is a complex Hilbert space and that closure and completeness are equivalent concepts.

TheOREM 1. If $\left\{\phi_{\gamma}(t) \psi_{\mu}(s)\right\}, \gamma, \mu=0,1, \cdots$, is a sequence of com-

${ }^{1}$ Presented to the Society, December 2, 1939.

${ }^{2}$ S. Saks, Theory of the Integral, English edition, p. 57.

${ }^{3}$ Curly brackets, \{\} , will always denote sequences. 
plex valued functions in $L_{2}\left(I_{2}\right)$, then a necessary and sufficient ${ }^{4}$ condition for closure is that $\left\{\phi_{\gamma}(t)\right\}$ and $\left\{\psi_{\mu}(s)\right\}$ be closed in the spaces $L_{2}\left(I_{t}\right)$ and $L_{2}\left(I_{s}\right)$ respectively.

We deal with the sufficiency demonstration first. Suppose the denumerable set of all subintervals, with rational end points, of $I_{t}$ to be ordered according to $0,1,2, \cdots$. We designate by $h_{\rho}(t)$ the characteristic function ${ }^{5}$ of the $\rho$ th subinterval divided by its norm. The function $g_{\nu}(s)$ is similarly defined for the range $I_{s}$. Thus

$$
\left\|h_{\rho}(t)\right\|_{I_{t}}=\left\|g_{\nu}(s)\right\|_{I_{s}}=1 .
$$

It is well known that $\left\{h_{\rho}(t) g_{\nu}(s)\right\}, \rho, \nu=0,1, \cdots$, has the closure property in $L_{2}\left(I_{2}\right)$. Hence for $f(s, t) \varepsilon L_{2}\left(I_{2}\right)$ and arbitrary $\epsilon>0$ we can find integers $M$ and $N$ and $M N$ complex constants $\left\{a_{\rho \nu}\right\}$ such that

$$
\left\|f(s, t)-\sum_{0}^{M} \sum_{0}^{N} a_{\rho \nu} h_{\rho}(t) g_{\nu}(s)\right\|<\epsilon / 2 .
$$

Let

$$
\delta \leqq \min \left(\frac{\epsilon}{4 M N} \max \left|a_{\rho \nu}\right|, 1\right)
$$

Thus

$$
2 \delta \sum_{0}^{M} \sum_{0}^{N}\left|a_{\rho \nu}\right|<\epsilon / 2 .
$$

In view of the assumed closure properties of $\left\{\phi_{\gamma}(t)\right\}$ and $\left\{\psi_{\mu}(s)\right\}$, integers $A$ and $B$ and complex constants $\left\{d_{\mu}^{(\nu)}\right\},\left\{e_{\gamma}^{(\rho)}\right\}, \rho=0,1, \cdots, M$ and $\nu=0,1, \cdots, N$, exist which yield the simultaneous inequalities

$$
\begin{aligned}
& \left\|g_{\nu}(s)-\sum_{\mu=0}^{B} d_{\mu}^{(\nu)} \psi_{\mu}(s)\right\|_{I}<\delta / 2, \\
& \left\|h_{\rho}(t)-\sum_{0}^{A} e_{\gamma}^{(\rho)} \phi_{\gamma}(t)\right\|_{I_{t}}<\delta / 2 .
\end{aligned}
$$

Hence

${ }^{4}$ A special case amounting to the assertion of sufficiency, only, for the subspace of $L_{2}\left(I_{2}\right)$ composed of real continuous functions, when $\left\{\phi_{\gamma}(t)\right\}$ and $\left\{\psi_{\mu}(s)\right\}$ are restricted to be orthogonal sets of functions, has been given by Courant: CourantHilbert, Methoden der mathematischen Physik, vol. 1, 1st edition, p. 90. Another special sufficiency proof is given in A. Zymund, Trigonometrical Series, p. 13.

${ }^{5}$ Saks, loc. cit., p. 6. 


$$
\left\|\sum_{0}^{B} d_{\mu}^{(\nu)} \psi_{\mu}(s)\right\|_{I_{s}} \leqq\left\|g_{\nu}(s)\right\|_{I_{t}}+\left\|g_{\nu}(s)-\sum_{0}^{B} d_{\mu}^{(\nu)} \psi_{\mu}(s)\right\|_{I_{s}} \leqq 2 .
$$

Let $\beta_{\gamma \mu}=\sum_{\rho=0}^{M} \sum_{\nu=0}^{N} a_{\rho \nu} e_{\gamma}^{(\rho)} d_{\mu}^{(\nu)}$. The triangle inequality for norms yields, in view of (6), (7), (7.11), and (7.2)

$$
\begin{aligned}
& \left\|h_{\rho}(t) g_{\nu}(s)-\sum_{0}^{A} \sum_{0}^{B} e_{\gamma}^{(\rho)} d_{\mu}^{(\nu)} \phi_{\gamma}(t) \psi_{\mu}(s)\right\| \\
& \leqq\left\|h_{\rho}(t)\left(g_{\nu}(s)-\sum_{0}^{B} d_{\mu}^{(\nu)} \psi_{\mu}(s)\right)\right\| \\
& +\left\|\sum_{0}^{B} d_{\mu}^{(\nu)} \psi_{\mu}(s)\left(h_{\rho}(t)-\sum_{c}^{A} e_{\gamma}^{(\rho)} \phi_{\gamma}(t)\right)\right\| \\
& \leqq\left\|h_{\rho}(t)\right\|_{I_{t}}\left\|g_{\nu}(s)-\sum_{0}^{B} d_{\mu}^{(\nu)} \psi_{\mu}(s)\right\|_{I_{s}} \\
& \quad+\left\|\sum_{0}^{B} d_{\mu}^{(\nu)} \psi_{\mu}(s)\right\| I_{I_{s}}\left\|h_{\rho}(t)-\sum_{0}^{A} e_{\gamma}^{(\rho)} \phi_{\gamma}(t)\right\|_{I_{t}} \\
& \leqq 2 \delta, \quad \text { for } \rho=0,1, \cdots, M, \nu=0,1, \cdots, N .
\end{aligned}
$$

On combining the various inequalities above

$$
\begin{aligned}
& \left\|f(s, t)-\sum_{0}^{A} \sum_{0}^{B} \beta_{\gamma \mu} \phi_{\gamma}(t) \psi_{\mu}(s)\right\| \\
& \leqq\left\|f(s, t)-\sum_{0}^{M} \sum_{0}^{N} a_{\rho \nu} h_{\rho}(t) g_{\nu}(s)\right\| \\
& \quad+\left\|\sum_{0}^{M} \sum_{0}^{N} a_{\rho \nu}\left(h_{\rho}(t) g_{\nu}(s)-\sum_{0}^{A} \sum_{0}^{B} e_{\gamma}^{\rho} d_{\mu}^{\nu} \phi_{\gamma}(t) \psi_{\mu}(s)\right)\right\| \\
& \leqq \epsilon / 2+\sum_{0}^{M} \sum_{0}^{N}\left(\left|a_{\rho \nu}\right|\left\|h_{\rho}(t) g_{\nu}(s)-\sum_{0}^{A} \sum_{0}^{B} e_{\gamma}^{(\rho)} d_{\mu}^{(\nu)} \phi_{\gamma}(t) \psi_{\mu}(s)\right\|\right) \\
& \leqq \epsilon / 2+2 \delta \sum_{0}^{M} \sum_{0}^{N}\left|a_{\rho \nu}\right| \leqq \epsilon .
\end{aligned}
$$

This asserts the closure property for $\left\{\phi_{\gamma}(t) \psi_{\mu}(s)\right\}$.

The necessity demonstration is equally direct. A trivial application of Fubini's theorem indicates that $\phi_{\gamma}(t) \varepsilon L_{2}\left(I_{t}\right), \psi_{\mu}(s) \varepsilon L_{2}\left(I_{s}\right)$ when $\phi_{\gamma}(t) \psi_{\mu}(s) \varepsilon L_{2}\left(I_{2}\right)$. No generality is lost if we assume that $\left\{\psi_{\mu}(s)\right\}$ is a linearly independent set of functions. Suppose $\left\{\psi_{\mu}(s)\right\}$ does not have the closure property. Then $f(s) \varepsilon L_{2}\left(I_{s}\right)$ exists for which for all $R$ and $b_{\mu}$ 


$$
\text { G.L.B. }\left\|f(s)-\sum_{0}^{R} b_{\mu} \psi_{\mu}(s)\right\|_{I_{s}}=c>0, \quad b_{\mu}=b_{\mu}^{\prime}+i b_{\mu}^{\prime \prime} \text {. }
$$

A fundamental result of Riesz guarantees the existence of minimal constants, ${ }^{6}\left\{b_{\mu}^{R}\right\}$, such that for $b_{\mu} \neq b_{\mu}^{R}, \mu \leqq R$,

$$
\left\|f(s)-\sum_{0}^{R} b_{\mu}^{R} \psi_{\mu}(s)\right\|_{I_{s}} \leqq\left\|f(s)-\sum_{0}^{R} b_{\mu} \psi_{\mu}(s)\right\|_{I_{s}} .
$$

The corresponding minimal constants for $A f(s)$ are evidently $\left\{A b_{\mu}^{R}\right\}$. Hence $^{7}$

$$
\left\|F(t) f(s)-\sum_{0}^{R} \bar{b}_{\mu}^{R} F(t) \psi_{\mu}(s)\right\|_{I_{s}} \leqq\left\|F(t) f(s)-\sum_{0}^{R} b_{\mu}(t) \psi_{\mu}(s)\right\|_{I_{\mathbf{s}}}, t \varepsilon I_{t},
$$

when $F(t) \varepsilon L_{2}\left(I_{t}\right)$ is a fixed function of positive norm. We write

$$
b_{\mu}(t)=\sum_{0}^{Q} a_{\gamma \mu} \phi_{\gamma}(t), \quad Q<\infty .
$$

In view of (12) we have

$$
\begin{aligned}
0<c\|F(t)\|_{I_{t}} & \leqq\left\|f(s) F(t)-\sum_{0}^{R} b_{\mu}^{R} F(t) \psi_{\mu}(s)\right\| \\
& =\left[\int_{I_{t}}\left\|f(s) F(t)-\sum_{0}^{R} b_{\mu}^{R} F(t) \psi_{\mu}(s)\right\|_{I_{s}}^{2} d I_{t}\right]^{1 / 2} \\
& \leqq\left[\int_{I_{t}}\left\|f(s) F(t)-\sum_{0}^{R} \sum_{0}^{Q} a_{\gamma \mu} \phi_{\gamma}(t) \psi_{\mu}(s)\right\|_{I_{s}}^{2} d I_{t}\right]^{1 / 2} \\
& =\left\|f(s) F(t)-\sum_{0}^{R} \sum_{0}^{Q} a_{\gamma \mu} \phi_{\gamma}(t) \psi_{\mu}(s)\right\| .
\end{aligned}
$$

Since (14) is in contradiction with the assumed closure property of $\left\{\phi_{\gamma}(t) \psi_{\mu}(s)\right\}$ our necessity proof is complete.

We denote by $h_{\rho}^{\prime}(t)$ and $g_{\nu}^{\prime}(s)$ the step functions in $R_{n}$ and $R_{m}$ analogous to $h_{\rho}(t)$ and $g_{\nu}(s)$. According to a classical result, $\left\{h_{\rho}^{\prime}(t) g_{\nu}^{\prime}(s)\right\}$, $\rho, \nu=0,1, \cdots$, have the closure property in $L_{2}\left(E_{2}\right)$ when the $s, t$ integration is over $R_{n+m}$ or any Lebesgue measurable subset $E_{2}$. Accordingly Theorem 1 and its demonstration remain formally valid in detail when $I_{s}, I_{t}$ and $I_{2}$ are replaced either by $R_{n}, R_{m}$ and $R_{n+m}$ or by

${ }^{6}$ F. Riesz, Acta Mathematica, vol. 41 (1916), p. 77, Lemma 3.

"With the choice $F(t) f(s)$, the method of proof of the necessity condition remains valid when sets of infinite measure are included. 
the sets $E_{s}, E_{t}$ and $E_{2}=E_{s} \times E_{t}$ of finite or infinite Lebesgue measure.

Theorem 2. If $\left\{F_{\rho}(s, t)\right\}, \rho=0,1, \cdots$, is closed in $L_{2}\left(E_{2}\right)$, then the sequence is also closed in $L_{2}\left(E_{s}\right)$ except possibly for a $t$ set of zero measure. $^{8}$

Suppose a lower bound of approximation to $f(s) \varepsilon L_{2}\left(E_{s}\right)$, by linear combinations of $\left\{F_{\rho}(s, t)\right\}$, is $c(t) \varepsilon L_{2}\left(E_{t}\right)$, where $\infty>c(t)>0$ for $t \varepsilon G \subset E_{t}$. Let $F(t) \varepsilon L_{2}\left(E_{t}\right)$ differ from 0 on $G$ (say $F(t)=c(t)$ ). The analogue of (14) is

$$
\|c(t) F(t)\|_{G} \leqq\|c(t) F(t)\|_{E_{t}} \leqq\left\|f(s) F(t)-\sum_{0}^{R} b_{\mu} F_{\mu}(s, t)\right\| .
$$

Hence $G$ has zero measure. Let $\left\{f^{(\sigma)}(s)\right\}$ be closed in $L_{2}\left(E_{s}\right)$ and denote the corresponding $G$ sets, defined above, by $\left\{G^{\sigma}\right\}$. The denumerable sum $\subseteq G^{\sigma}$ is plainly of measure zero. Thus $\left\{F_{\rho}(s, t)\right\}$ is closed in $L_{2}\left(E_{s}\right)$ for all $t \varepsilon E_{t}-\Im G^{\sigma}$.

We now abstract the properties needed in the foregoing proofs. Let $T(E)$ denote a Banach space ${ }^{9}$ of real functions on $E$. A set $G, G \subset E$, will be called a non-significant set if $f(z) \varepsilon T(E)$ may be arbitrarily changed on $G$ without affecting the value of $\|f(z)\|_{E}$. The postulates below hold for $T(E)$. When (d) and (e) are omitted we write $T_{-}(E)$.

(a) If $f(s, t) \varepsilon T\left(E_{2}\right)$ then $f(s, t) \varepsilon T\left(E_{s}\right)$ and $f(s, t) \varepsilon T\left(E_{t}\right)$ for all save a non-significant set of $t$ or $s$ values respectively. If $f(s) \varepsilon T\left(E_{s}\right)$, $F(t) \varepsilon T\left(E_{t}\right)$ then $f(s) F(t) \varepsilon T\left(E_{2}\right)$.

(b) $\|f(s, t)\|_{E_{2}}=\|\| f(s, t)\left\|_{E_{s}}\right\|_{E_{t}}$.

(c) If, neglecting non-significant sets, $\left|f_{1}(t)\right|>\left|f_{2}(t)\right|$, then $\left\|f_{1}(t)\right\|_{E_{t}}$ $>\left\|f_{2}(t)\right\|_{E_{t}}$.

(d) There exists a sequence $\left\{h_{\rho}(t) g_{\nu}(s)\right\}, \rho, \nu=0,1, \cdots$, with the closure property in $T\left(E_{2}\right)$, where $h_{\rho}(t) \varepsilon T\left(E_{t}\right)$ and $g_{\nu}(s) \varepsilon T\left(E_{s}\right)$.

(e) Denumerable sums of non-significant sets are non-significant sets.

${ }^{8}$ A sharper result follows from Fatou's lemma. Suppose $F(t) \varepsilon L_{2}\left(E_{t}\right)$ differs from 0 for almost all $t \varepsilon E_{t}$. Now

$$
0=\mathrm{L}_{N \rightarrow \infty}\left\|f(s) F(t)-\sum_{0}^{N} b_{\rho}^{(N)} F_{\rho}(s, t)\right\|_{E_{2}}^{2} \geqq \int_{E_{t}} \mathrm{~L}_{N \rightarrow \infty}\left\|f(s) F(t)-\sum^{N} b_{\rho}^{(N)} F_{\rho}(s, t)\right\|_{E_{s}}^{2} d E_{t} .
$$

Thus a suitable sequence $\left\{\sum^{N_{i} b_{\rho}^{(i)}} F_{\rho}(s, t)\right\}$, with constant coefficients $\left\{b_{\rho}^{(i)}\right\}$, converges strongly to $f(s)$ in $L_{2}\left(E_{s}\right)$ for almost all $t \varepsilon E_{t}$. Moreover if $E_{t}$ is of finite measure, the Egoroff theorem guarantees uniform convergence for $t \varepsilon D_{\delta} \subset E_{t}$ where the measure of $E_{t}-D_{\delta}$ is inferior to arbitrary $\delta$. A closed sequence $\left\{f_{\sigma}(s)\right\}$ is introduced as above.

$\checkmark \mathrm{S}$. Banach, Théorie des Opérations Linéaires, pp. 53, 58. Banach uses fundamental in the sense of our closed. 
Theorem 3. $(\alpha)$ If $\left\{\phi_{\gamma}(t)\right\}$ and $\left\{\psi_{\mu}(s)\right\}$ are closed in $T\left(E_{t}\right), T\left(E_{s}\right)$ then $\left\{\phi_{\gamma}(t) \psi_{\mu}(s)\right\}$ is closed in $T\left(E_{2}\right) .(\beta)$ If $\left\{\phi_{\gamma}(t) \psi_{\mu}(s)\right\}$ is closed in $T_{-}\left(E_{2}\right)$, then $\left\{\psi_{\mu}(s)\right\}$ is closed in $T_{-}\left(E_{s}\right) .(\gamma)$ If $\left\{F_{\rho}(s, t)\right\}$ is closed in $T\left(E_{2}\right)$, then $\left\{F_{\rho}(s, t)\right\}$ is closed in $T\left(E_{s}\right)$ for all but a non-significant set of $t$ values in $E_{t}$.

The demonstrations of Theorems 1 and 2 apply without change in form. ${ }^{10}$ The space $^{11} L_{p}(E, \mu), p \geqq 1$, is included in $T(E)$. This is the space of measurable functions whose $p$ th powers are summable over the measurable set $E$, where the Lebesgue-Radon-Stieltjes integral is equally admissible with the usual Lebesgue integral. Thus the symbol $\mu(E)$ denotes either the Lebesgue measure, or the Radon measure determined by a non-negative additive function of intervals. In all cases $\mu_{2}\left(E_{2}\right)=\mu_{s}\left(E_{s}\right) \mu_{t}\left(E_{t}\right)$, and the sets of zero measure constitute the non-significant sets. The norm is

$$
\|f(s, t)\|=\left[\int_{E}|f|^{p} d \mu(E)\right]^{1 / p} .
$$

The verification of the main postulates is implied by the Fubini theorem, the Hölder-Minkowski inequalities and the denseness of the step functions. The functions $\left\{h_{\rho}(t)\right\},\left\{g_{\nu}(s)\right\}$ or $\left\{h_{\rho}^{\prime}(t) g_{\nu}^{\prime}(s)\right\}$ as defined in Theorem 1 are again available. ${ }^{12}$

The space $C(E)$ of continuous functions is another special case of $T(E)$. We assume $E_{s} \leqq R_{m}, E_{t} \leqq R_{n}$ and $E_{2} \leqq R_{n+m}$ are bounded closed sets. The null set is the only non-significant set. The norm is

$$
\|f(s, t)\|=\max _{s, t \varepsilon E_{2}}|f(s, t)| .
$$

The sequences $h_{\rho}(t)$ and $g_{\nu}(s)$ are the ordered products of the elements $1, t_{1}, \cdots, t_{n}$ and of $1, s_{1}, \cdots, s_{m}$ respectively.

Postulates (b) and (c) may be replaced by the weaker

$\left(\mathrm{b}^{\prime}\right)\|f(w, z)\|_{E_{2}}<\epsilon$ implies $\|f(w, z)\|_{E_{w}}<\eta(\epsilon)$, where $\mathrm{L}_{\epsilon \rightarrow 0} \eta(\epsilon)=0$ except possibly for non-significant $z$ sets.

$\left(\mathrm{c}^{\prime}\right)\|G(z)\|_{E_{z}}=1,\|H(w)\|_{E_{w}}<\epsilon$ imply $\|G(z) H(w)\|_{E_{2}}<\eta(\epsilon)$.

${ }^{10}$ For $(\alpha)$, postulate (d) may be replaced by the assumption that each $f(s, t) \varepsilon T\left(E_{2}\right)$ is the strong limit of some (not necessarily fixed) sequence $\left\{h_{\rho}^{f}(t) g_{\nu}^{f}(s)\right\}$, where $h_{\rho}^{f}(t) \varepsilon T\left(E_{t}\right)$ and $g_{\nu}^{f}(s) \varepsilon T\left(E_{s}\right)$.

${ }^{11}$ Saks, loc. cit. (1928), chap. 3, or J. Radon, Sitzungsberichte der Akademie der Wissenschaften, Vienna, class IIa, vol. 122 (1913). The Lebesgue case admits sets of infinite measure.

${ }^{12}$ For $p>1$ a valid theorem on completeness is obtained from Theorem 3 if closure (in $L_{p}(E, \mu)$ ) is replaced by completeness in $L_{p /(p-1)}(E, \mu)$ where $E$ refers to $E_{s}, E_{t}$ and $E_{2}$ in turn. 
These modifications will be connoted by writing $T^{\prime}(E)$ and $T^{\prime}(E)$. Consider, for instance, $C^{1}(E)$, the space of functions continuous together with their first partial derivatives on ${ }^{13} E$. We restrict ourselves now to closed linear intervals $I_{s}, I_{t}$ and the rectangle $I_{2}: I_{s} \times I_{t}$. The norms in $C^{1}\left(I_{2}\right)$ and $C^{1}\left(I_{s}\right)$ are, ${ }^{14}$ with $f_{s} \equiv \partial f / \partial s$,

$$
\begin{aligned}
\|f(s, t)\| & =\max _{I_{2}}|f(s, t)|+\max _{I_{2}}\left|f_{s}(s, t)\right|+\max \left|f_{t}(s, t)\right|, \\
\|f(s)\| & =\max _{I_{s}}|f(s)|+\max _{I_{s}}\left|f_{s}(s)\right| .
\end{aligned}
$$

It is well known that $C^{1}\left(I_{s}\right)$ (and $C^{1}\left(I_{t}\right)$ ) is complete. It is easy to show that $C^{1}\left(I_{2}\right)$ also is complete. Indeed if $\left\{f^{(n)}(s, t)\right\}$ is a Cauchy sequence in $C^{1}\left(I_{2}\right)$, then $f^{(n)}(s, t), f_{s}^{(n)}(s, t)$ and $f_{t}^{(n)}(s, t)$ converge $u n i$ formly in $I_{2}$ and hence define an element of $C^{1}\left(I_{2}\right)$.

Since

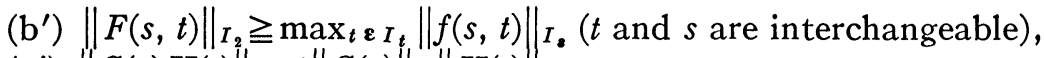

(c') $\|G(s) H(t)\|_{I_{2}} \leqq\|G(s)\|_{I_{s}}\|H(t)\|_{I_{t}}$.

it is clear that $\left(b^{\prime}\right)$ and $\left(c^{\prime}\right)$ are satisfied.

THEOREM 4. The conclusions in $(\alpha),(\beta),(\gamma)$ of Theorem 3 remain valid when $T^{\prime}(E)$ and $T_{-}^{\prime}(E)$ replace $T(E)$ and $T_{-}(E)$.

For $(\alpha)$ we now choose $\delta$ small enough in (7) and (7.1) to yield $\eta(\delta)$ inferior to the right side of (6). Then (6.1) is valid with $\eta(\delta)$ written in place of $\delta$. On making use of $\left(c^{\prime}\right)$ it is easily shown that the left side of $(8)$ is smaller than $2 \eta(\delta)$ and the final inequality in (9) is again obtained. For $(\beta)$ we need only change (14) slightly. Indeed, by reference to $\left(b^{\prime}\right)$ and (13)

$$
\epsilon \geqq\left\|f(s) F(t)-\sum \sum a_{\gamma \mu} \phi_{\gamma}(t) \psi_{\mu}(s)\right\|_{I_{2}}
$$

would imply the contradiction

$$
\eta(\epsilon) \geqq|c| \text { true } \max |F(t)|>0 .
$$

The true maximum is defined just as in the analogous case of measurable functions and implies neglect of non-significant $t$ sets. Evidently $(\gamma)$ also may be maintained. Indeed the argument in footnote 8 , for ex , ple, is easily amended to yield the desired result.

If the closure property of the sequence $\left\{\phi_{p}(z)\right\}$ in $T_{-}(E)$ or $C^{1}(I)$ is unaffected by the omission of $\phi_{\sigma}(z)$, then we shall say $\left\{\phi_{\rho}(z)\right\}$ is a

\footnotetext{
${ }^{13}$ The sets used in $C(E)$ are available for $C^{1}(E)$ also.

${ }^{14}$ Even if $f(s, t)$ and $g(s) h(t) \varepsilon C^{1}\left(E_{2}\right),\|\| f(s, t)\left\|_{I_{s}}\right\|_{I_{t}}$ and \|\|$g(s) h(t)\|\|_{I_{s}} \|_{I_{t}}$ need not exist. Thus $C^{1}(E)$ is not included under $T(E)$.
} 
"redundant" sequence and $\phi_{\sigma}(z)$ is a "superfluous" function. If $\left\{\phi_{\sigma_{k}}\right\}$, $k=1,2, \cdots, K$, is superfluous, then for arbitrary $\epsilon$ we can satisfy

$$
\left\|\phi_{\sigma_{k}}-\sum_{0}^{N} c_{j} \phi_{j}(z)\right\|<\epsilon, \quad j \neq \sigma_{l}, l=1, \cdots, K .
$$

Lemma 1. In $T_{-}(E)$ or $T_{-}^{\prime}(E)$ if $\left\{f_{\mu}(z)\right\}$ is closed and non-redundant, then for any $F(z), \overline{\mathrm{L}}_{\epsilon \rightarrow 0}\left|d_{1}(\epsilon)\right| \leqq D<\infty$ where $d_{1}(\epsilon)$ is consistent with $\left\|F(z)-d_{1}(\epsilon) f_{1}(z)-\sum_{2}^{N} d_{j} f_{j}(z)\right\|<\epsilon$.

In the contrary case

Now

$$
\begin{aligned}
\epsilon+\|F(z)\| & \geqq\|F(z)\|+\left\|F(z)-d_{1}(\epsilon) f_{1}(z)-\sum_{2}^{N} d_{i} f_{j}(z)\right\| \\
& \geqq\left|d_{1}(\epsilon)\right|\left\|f_{1}(z)-\sum_{2}^{N} \frac{d_{i}}{d_{1}} f_{i}(z)\right\| .
\end{aligned}
$$

$$
\left\|f_{1}(z)-\sum_{2}^{N} \frac{d_{i}}{d_{1}} f_{i}(z)\right\| \geqq c>0,
$$

for all $N$ and $d_{i}$, since $f_{1}(z)$ is not superfluous. For all sufficiently small $\epsilon,(19)$ and (20) imply

$$
\left|d_{1}(\epsilon)\right| \leqq 2\|F(z)\| / c
$$

in contradiction with the hypothesized non-boundedness of $d_{1}(\epsilon)$.

TheOREM 5. If $\left\{\phi_{\mu}(t) \psi_{\mu}(s)\right\}$ is closed in $T_{-}\left(E_{2}\right)$ or $C^{1} E$, then (I) $\left\{\psi_{\mu}(s)\right\}$ is closed in $T_{-}\left(E_{s}\right)\left(\right.$ or $\left.C^{1}\left(I_{s}\right)\right)$; (II) every finite subsequence of $\left\{\psi_{\mu}(s)\right\}$ is superfluous. ${ }^{15}$

Evidently (I) is a special case of Theorem $3(\beta)$. In view of (I) if $\phi_{\sigma}(T) \psi_{\sigma}(s), \sigma=1, \cdots, q$, is superfluous, then $\psi_{\sigma}(s), \sigma=1, \cdots, q$, is superfluous. Accordingly we may restrict ourselves to non-redundant sequences $\left\{\phi_{\mu}(t) \psi_{\mu}(s)\right\}$.

We demonstrate (II) by induction. Suppose $\psi_{1}(s), \cdots, \psi_{n-1}(s)$ are superfluous. Since no finite basis exists in $T_{-}(E)$ or $C^{1}(I)$, we may find a function $F(t)$ such that the set $F(t), \phi_{\sigma}(t), \sigma=1, \cdots, n$, is linearly independent. Suppose $\psi_{n}(s)$ is not superfluous. Then

$$
\left\|\psi_{n}(s)-\sum_{n+1}^{N} k_{i} \psi_{i}(s)\right\|_{E} \geqq c>0
$$

${ }^{15}$ Evidently $\left\{\psi_{\mu}(s)\right\}$ need not be dense closed in the sense that any infinite subsequence is closed. 
for all $k_{i}$ and $N$. By hypothesis sequences $\left\{a_{i}^{(\rho)}\right\}$ and a constant $N$ exist for arbitrary $\epsilon$ such that

$$
\left\|\psi_{\rho}(s)-\sum_{i=n}^{N} a_{i}^{(\rho)} \psi_{i}(s)\right\|_{E_{s}} \leqq \epsilon, \quad \rho=1, \cdots, n-1 .
$$

Moreover

$$
\begin{aligned}
\| \psi_{n}(s) F(t)- & \sum_{\rho=1}^{n} d_{\rho} \phi_{\rho}(t) \psi_{\rho}(s)-\sum_{n+1}^{N} d_{i} \phi_{i}(t) \psi_{i}(s) \| \\
& +\left\|\sum_{\rho=1}^{n-1} d_{\rho} \phi_{\rho}(t)\left(\psi_{\rho}(s)-\sum_{n}^{N} a_{i}^{(\rho)} \psi_{i}(s)\right)\right\| \\
\geqq & \| \psi_{n}(s)\left[F(t)-\sum_{\rho=1}^{n-1} d_{\rho} a_{n}^{\rho} \phi_{\rho}(t)-d_{n} \phi_{n}(t)\right] \\
& -\sum_{n+1}^{N} d_{i} \phi_{i}(t) \psi_{i}(s)-\sum_{\sigma=1}^{n-1} \sum_{n+1}^{N} a_{i}^{(\sigma)} d_{\sigma} \phi_{\sigma}(t) \psi_{i}(s) \| .
\end{aligned}
$$

The right side of this inequality, by an argument similar in all details to that involved in the passage from (12) to (14), dominates

$$
c\left\|F(t)-\sum_{1}^{n-1} d_{\sigma} a_{n}^{(\sigma)} \phi_{\sigma}(t)-d_{n} \phi_{n}(t)\right\|_{I_{t}} \text { in } T_{-}\left(E_{2}\right)
$$

or $\left(c f .\left(b^{\prime \prime}\right)\right)$

$$
\max _{t \varepsilon I_{t}} c\left|F(t)-\sum_{1}^{n-1} d_{\sigma} a_{n}^{\sigma} \phi_{\sigma}(t)-d_{n} \phi_{n}(t)\right| \text { in } C^{1}\left(I_{2}\right) .
$$

In (23.2) we note $\phi_{j}(t) \varepsilon C^{1}\left(I_{t}\right)$ implies $\phi_{j}(t) \varepsilon C\left(I_{t}\right)$. Hence again by the Riesz theorem the expressions in (23.1) and (23.2) have a positive lower bound, denoted by $K>0$. In view of (22.1) closure of $\left\{\phi_{\mu}(t) \psi_{\mu}(s)\right\}$ and postulates (b) or $\left(\mathrm{c}^{\prime \prime}\right)$, constants $N, d_{i}$ and $a_{i}^{(\rho)}$ exist such that the left side of (23) is inferior to

$$
\epsilon+\sum_{1}^{n-1}\left|d_{\rho}\right|\left\|\phi_{\rho}(t)\right\|_{I_{t} \epsilon}
$$

Hence by Lemma 1 applied to each $d_{\rho}$ the upper bound in (24) approaches 0 with $\epsilon$ in contradiction with the conclusion $K>0$. Thus $\psi_{n}(s)$ is superfluous.

This type of argument may be used to show that the non-redundancy of $\left\{\phi_{\mu}(t) \psi_{\mu}(s)\right\}$ implies that $\psi_{1}(s)$ is superfluous. The induction is thus complete and part (II) of our theorem is established. It is an easy matter to extend the theorem to $T_{-}^{\prime}(E)$ spaces.

UNIVERSITY OF ILLINOIS 